recorded prevalence of comorbidities potentially linked to chest pain in patients with NCCP in primary care; and (ii) determine the incidence of future CVD in those with NCCP. Methods A cohort study was conducted between 2002-2015 using the Consultations in Primary Care Archive (CiPCA) database of pseudonymised primary care data. Patients aged $>18$ years with no prior CVD presenting with NCCP (index date) were included. Recorded comorbidities (osteoarthritis [OA]; lower back pain [LBP]; depression/anxiety; respiratory; reflux; and cancer) were identified between 24 months prior to and 6 months after the index date of NCCP. NCCP was categorised into those recorded with a non-cardiac cause at index date, unattributed chest pain with no comorbidity, and unattributed chest pain with comorbidity. Variation in patient characteristics were investigated. Incidence rates (IRs) of CVD events per 1,000 person years were calculated by NCCP category and stratified by time windows from the index date to CVD.

Results In total, 20,996 patients presented with NCCP with $9,000(43 \%)$ recorded with a non-cardiac cause at index date, 5,993 (28\%) unattributed no comorbidity, and 6,003 (29\%) unattributed with comorbidity. The non-cardiac cause NCCP group were younger (44.1 versus 44.3 and 49.3 years) with a lower proportion of men (41.6\% versus $42.4 \%$ and $54.1 \%)$ compared to the unattributed no comorbidity and unattributed with comorbidity groups, respectively. The most common comorbidity was respiratory (35\%) followed by depression/ anxiety (33\%), reflux (15\%), LBP (8\%), OA (6\%) and cancer $(3 \%)$ in the unattributed with comorbidity group. The unattributed with comorbidity group had the highest incidence of CVD (IR 16.3, 95\% confidence interval [CI] 15.0-17.6) vs. non-cardiac cause NCCP (IR 8.8, 95\% CI 8.1-9.6) and unattributed no comorbidity groups (IR 11.7, 95\% CI 10.7-12.8). The highest incidence of CVD occurred between 6-12 months after the index date (IR 45.0, 95\% CI 32.7-61.8). Patients in the unattributed with comorbidity group with OA (IR 29.2, 95\% CI 23.4-36.4) and cancer (IR 29.2, 95\% CI 20.0-42.6) had the highest incidence of CVD, reflecting their older age. Conclusions Depression and respiratory symptoms may be the most common reasons for NCCP in primary care. A higher incidence of CVD was observed for patients with unattributed NCCP and comorbidity, and in the first 6-12 months after presentation to primary care. This study highlights the importance of following up patients with unattributed chest pain symptoms.

Conflict of Interest N/A

\section{ASSOCIATION BETWEEN CAROTID DISTENSIBILITY AND HEART RATE VARIABILITY IN OLDER AGE}

${ }^{1}$ Massimiliano Fornasiero, ${ }^{1}$ Matt Webber, ${ }^{2}$ James Moon, ${ }^{3}$ Peter Friberg, ${ }^{4}$ Alun Hughes, ${ }^{5}$ Gaby Captur. ${ }^{1}$ Institute of Cardiovascular Science, University College London, London, UK; ${ }^{2}$ Barts Health NHS Trust; ${ }^{3}$ Department of public health and community medicine, Institute of Medicine, Sahlgrenska Academy; ${ }^{4}$ mperial College London; ${ }^{5}$ The Royal Free Hospital, Centre for Inherited Heart Muscle Conditions

\subsection{6/heartjnl-2021-BCS.187}

Background Heart rate variability (HRV) is a prognostically relevant biomarker of autonomic regulation. Carotid stiffening that may precede hypertension, could theoretically inhibit the baroreceptor reflex, resulting in cardiac autonomic dysfunction and abnormal HRV indices. We investigated the association between carotid artery distensibility (the inverse of carotid arterial stiffness) and HRV in an older age population-based cohort.

Method Participants were from the 1946 Medical Research Council (MRC) National Survey of Health and Development (NSHD) British birth cohort. Carotid intima media thickness (cIMT) and carotid artery distensibility (exposures) were measured by ultrasound. Calibrated images and dedicated software automatically measured carotid distensibility, while semi-automatic edge detection software was used to measure cIMT. Time- and frequency-domain analysis of each participant's 6minute resting 3-lead tachogram at age 60-64 years yielded the following HRV biomarkers (outcomes): standard deviation of normal-to-normal beats (SDNN); root mean square of successive differences (RMSDD); HRV triangular index; high-frequency (HF) power; low-frequency (LF) power; total power spectral density (PSD); power spectral density squared; normalised LF and HF power and HF:LF ratio. Generalized linear models (glm) were used, and adjustment was made for relevant demographic and health-related confounders including BMI, sex socio-economic position and plasma triglyceride levels.

Results A total of 896 participants were included $(46.5 \%$ men, figure 1). On univariate analysis carotid distensibility, but not cIMT, correlated with the following HRV markers: SDNN, total PSD and HRV triangular index (markers of overall variability), RMSDD (marker of parasympathetic activity), HF power and LF power (markers of parasympathetic and sympathetic activity, all $\mathrm{p}<0.05)$. These associations persisted in fully adjusted multivariable models (table 1): SDNN beta 0.63 ; $95 \%$ confidence interval (CI) $0.4,0.9 ; \mathrm{p}<0.001$; RMSDD beta 0.60 ; CI $0.3,0.9 ; \mathrm{p}<0.001$; HRV triangular index; beta 0.13 ;

Abstract 190 Table 1 Multivariable regression analysis. Showing results of multivariable regression analysis separately for each of the HRV biomarkers with exposure variables (including carotid distensibility) that were significant at univariate analysis $(p<0.05)$. Some boxes, for example in hypertension, do not have data because they were not significant at univariate analysis for the relevant HRV outcome. SDNN = standard deviation of normal-to-normal beats. RMSDD = root mean square of successive differences. HRV = heart rate variability. HF = high-frequency. LF = low-frequency. $\mathrm{Cl}$ = confidence interval. $\mathrm{BMI}=$ body mass index. $\mathrm{MI}$ = myocardial infarction. $\mathrm{SEP}=$ socio-economic position

\begin{tabular}{|c|c|c|c|c|c|c|c|c|c|c|c|c|}
\hline \multirow[b]{2}{*}{ Variable } & \multicolumn{2}{|l|}{ SDNN } & \multicolumn{2}{|c|}{ RMSDD } & \multicolumn{2}{|c|}{ HRV Triangular index } & \multicolumn{2}{|l|}{ HF power } & \multicolumn{2}{|l|}{ Lf power } & \multicolumn{2}{|c|}{ Total power spectral density } \\
\hline & $\begin{array}{l}\text { B Coefficient } \\
(95 \% \mathrm{Cl})\end{array}$ & p-value & $\begin{array}{c}\text { B Coefficient } \\
(95 \% \mathrm{Cl})\end{array}$ & $\mathrm{p}$-value & $\begin{array}{c}\beta \text { Coefficient } \\
(95 \% \mathrm{Cl})\end{array}$ & $\mathrm{p}$-value & $\begin{array}{c}\beta \text { Coefficient (95\% } \\
\mathrm{Cl})\end{array}$ & $\begin{array}{c}\mathrm{p}- \\
\text { value }\end{array}$ & $\beta$ Coefficient $(95 \% \mathrm{Cl})$ & p-value & $\beta$ Coefficient $(95 \% \mathrm{Cl})$ & p-value \\
\hline $\begin{array}{c}\text { Carotid } \\
\text { distensibility }\end{array}$ & $0.63(0.4,0.9)$ & $<0.001^{*}$ & $0.60(0.3,0.9)$ & $<0.001^{*}$ & $0.13(0.1,0.2)$ & $<0.001^{*}$ & $8.04(2.3,13.8)$ & $0.006^{*}$ & $9.86(4.7,17.0)$ & $0.012^{*}$ & $25.40(8.8,43.4)$ & $0.008^{*}$ \\
\hline Sex & $-2.97(-5.1,-0.9)$ & $0.005^{*}$ & $-0.04(-1.9,1.9)$ & 0.966 & $-0.31(-0.8,0.1)$ & 0.165 & $21.45(-22.3,65.2)$ & 0.336 & $-23.04(-84.7,48.3)$ & 0.474 & $-135.00(-273.0,-2.5)$ & $0.045^{*}$ \\
\hline BMI & $-0.02(-0.3,0.2)$ & 0.857 & $0.11(-0.1,0.4)$ & 0.338 & $-0.02(-0.1,0.03)$ & 0.419 & $4.81(-0.5,10.1)$ & 0.076 & $0.15(-5.8,7.0)$ & 0.962 & $3.43(-11.7,19.9)$ & 0.671 \\
\hline SEP 1989 & $-0.08(-2.6,2.6)$ & 0.950 & $-0.56(-2.8,1.8)$ & 0.631 & $-0.25(-0.8,0.3)$ & 0.372 & $-21.10(-76.1,33.9)$ & 0.452 & $-23.04(-84.7,48.3)$ & 0.474 & $2.49(-153.8,179.0)$ & 0.976 \\
\hline Triglyceride & $-1.32(-2.8,0.3)$ & 0.078 & $-1.46(-2.6,-0.2)$ & $0.017^{*}$ & $-0.26(-0.6,0.1)$ & 0.101 & $-38.26(-69.6,-6.9)$ & $0.017^{*}$ & $-36.83(-61.8,4.7)$ & $0.023^{*}$ & $-86.01(-162.9,13.3)$ & $0.043^{*}$ \\
\hline HBAIC & $-0.12(-0.2,0.01)$ & 0.053 & & & $-0.03(-0.1,-0.004)$ & $0.019^{*}$ & & & $-1.88(-3.7,1.1)$ & 0.135 & $-5.70(-10.7,2.2)$ & 0.079 \\
\hline Ml or angina & $-3.62(-7.5,0.9)$ & 0.085 & & & & & & & & & & \\
\hline Stroke & & & $-1.12(-1.6,-0.1)$ & $0.001^{*}$ & $-0.22(-0.4,0.03)$ & $0.033^{*}$ & $-15.49(-43.2,12.2)$ & 0.272 & $-18.15(-29.6,9.9)$ & $<0.001^{*}$ & $-56.78(-86.9,-0.4)$ & $<0.001^{*}$ \\
\hline Hypertension & & & $-1.27(-3.2,0.7)$ & 0.191 & & & & & & & & \\
\hline
\end{tabular}




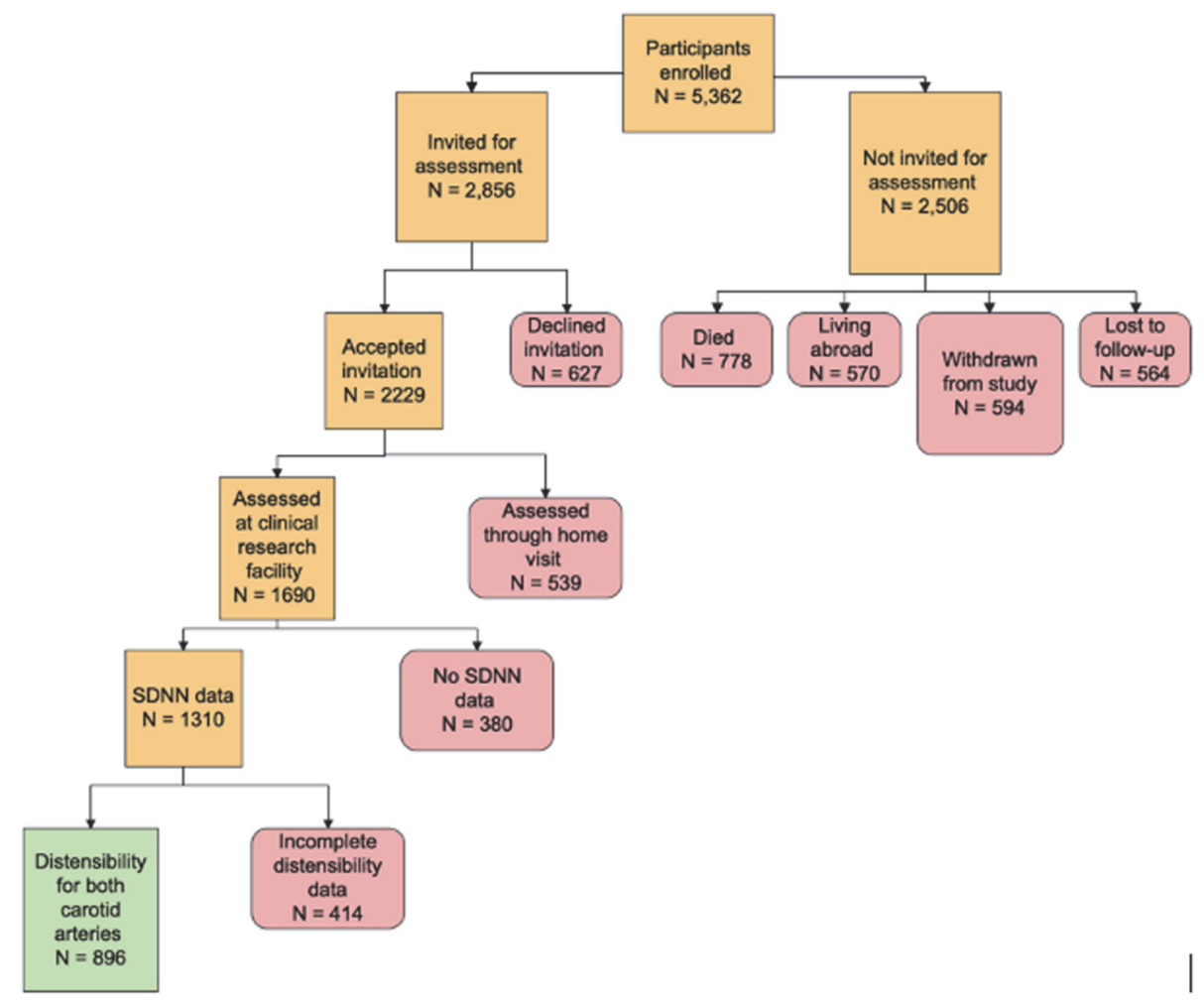

Abstract 190 Figure 1 Study flowchart. Flowchart summarising participants that were excluded or lost to follow-up, and how the final study sample was derived. SDNN =standard deviation of normal-to-normal beats.

CI $0.06,0.19 ; \mathrm{p}<0.001 ; \mathrm{HF}$ power; beta $8.04 ; \mathrm{CI} 2.3,13.8$; $\mathrm{p}=0.006$; LF power beta 9.86; CI 4.7,17.0; $\mathrm{p}<0.012$; total PSD beta 25.40; CI 8.8,43.4; $\mathrm{p}=0.008$.

Conclusion Carotid arterial stiffness in older age is associated with reduced HRV independent of early carotid atherosclerosis (cIMT), hypertension or stroke, potentially through an impaired baroreceptor response. As loss of carotid distensibility can be prevented and loss of HRV predicts cardiovascular mortality, public health policies should implement more and earlier cardiovascular health interventions to maintain carotid distensibility, with HRV as a marker of disease progression.

Conflict of Interest None

\section{ASSOCIATIONS OF NON-ALCOHOLIC FATTY LIVER DISEASE WITH SUBCLINICAL ATHEROSCLEROSIS AND ECHOCARDIOGRAPHY MEASUREMENTS IN YOUNG ADULTS}

${ }^{1}$ Rosalind Tang, ${ }^{1}$ Kushala Abeysekera, 'Laura Howe, ${ }^{2}$ Alun Hughes, ${ }^{1}$ Abigail Fraser. ${ }^{1}$ University of Bristol, Bristol, UK; ${ }^{2}$ Imperial College London

\subsection{6/heartjnl-2021-BCS.188}

Background Non-alcoholic fatty liver disease (NAFLD) is becoming increasingly prevalent worldwide, even among young adults. This condition shares many risk factors with other metabolic disorders, including cardiovascular disease (CVD), but researchers suggest the presence of NAFLD itself may be a risk factor for developing CVD, independently of other established factors.

Methods In this prospective, general population-based cohort study of young adults in the UK, transient elastographydefined liver steatosis and fibrosis were assessed at age 24 years, as were cardiovascular structure and function, measured using echocardiography, carotid ultrasonography and pulse wave analysis. We examined associations between liver and cardiovascular health, with and without accounting for age, sex, ethnicity, social class, employment, body mass index, alcohol, smoking, blood pressure, fasting serum lipids, fasting glucose, fasting insulin and C-reactive protein. All participants with harmful alcohol consumption or viral hepatitis were excluded from analysis. Pregnant participants were also excluded from ultrasonography and therefore could not be included.

Results A total of 2,047 young adults (mean age 24.4 years; $36.2 \%$ female) from the Avon Longitudinal Study of Parents and Children (ALSPAC) were included; 406 (19.8\%) had liver steatosis, while 38 (1.9\%) had liver fibrosis F2 or greater. After full-adjustment for established cardiovascular risk factors, steatosis was only associated with a decrease in stroke volume index (adjusted mean difference [95\% CI] of -1.21 [-2.14, $0.10] \mathrm{mL} / \mathrm{m} 2$ ). All other associations of steatosis with cardiovascular outcomes were null in our fully-adjusted model. Liver fibrosis, however, was associated with several measures of cardiac structure and function, as well as subclinical atherosclerosis. Compared to F0/F1 fibrosis without septa, presence of F2-F4 fibrosis was associated with lower cardiac output index (adjusted mean difference [95\% CI] of $-0.22[-0.39,-0.05] \mathrm{L} /$ $\mathrm{min} / \mathrm{m} 2$ ) and with greater tricuspid annular plane systolic excursion (adjusted mean difference [95\% CI] of $0.13[0.01$, $0.25] \mathrm{cm}$ ), left ventricular mass index (adjusted mean difference $[95 \% \mathrm{CI}$ ] of $2.02[0.06,3.99] \mathrm{g} / \mathrm{m} 2.7)$, mitral valve E/A ratio (adjusted mean difference $\left[\begin{array}{lllll}95 \% & \mathrm{CI}\end{array}\right]$ of $0.30[0.12$, $0.48]$ ), carotid intima-media thickness (adjusted mean difference $[95 \% \mathrm{CI}]$ of $0.02[0.01,0.04] \mathrm{mm})$ and carotid-femoral pulse wave velocity (adjusted mean difference [95\% CI] of $0.42[0.08,0.76] \mathrm{m} / \mathrm{s}$ ) after full-adjustment for covariates. 